\title{
Temperature Changes Effect on the Inner Product Vector Method and its Application to Structural Health Monitoring of Aircraft Industry
}

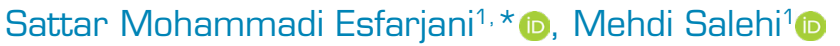 \\ 1.Islamic Azad University - Najafabad Branch - Department of Mechanical Engineering - Najafabad - Iran. \\ *Corresponding author: satar.iran@gmail.com
}

\begin{abstract}
The purpose of the paper is to study the effect of temperature change on the theory of inner product vector (IPV). The IPV method can be used to detect structural damage. This study evaluates the IPV method ability to detect damage of an Airbus A320 slat-track, which is in the form of a longitudinal crack. The results show that the IPV method is able to detect defects in the structure as well as its location, with close approximation. Then, the Airbus A320 slat-track was investigated for the effect of changes in temperature on the IPV method, evaluated over a temperature between -73 and $260^{\circ} \mathrm{C}$. The effect of temperature on the performance of IPV damage detection method has not been investigated so far. The results of the IPV method show a spurious defect in the structure as the temperature changes; therefore, the IPV method is temperature-sensitive. Also, this study highlighted the importance of applying simulation methods to develop vibration-based damage detection (VBDD) techniques, especially for evaluating the effect of changes in environmental temperature when the structure is complex.
\end{abstract}

Keywords: Vibration-Based Damage Detection; Cross-correlation function; Temperature; Airbus 320 slat-track; Finite element.

\section{INTRODUCTION}

Nowadays the topic of vibration-based structural health monitoring (SHM) methods has been widely used in structural damage detection. Vibration-based techniques can be classified into two categories: frequency domain methods and time domain methods (Labib et al. 2014; Medeiros et al. 2013; Ni et al. 2014). The use of cross-correlation functions (CFF) is one of the time domain methods that have attracted many researchers in recent years and has been implemented in some practical applications in SHM.

Farrar and James (1997) proved that the CCF of the responses can be adopted for recognizing structural dynamic properties. The Hilbert-Huang transform of the CCF to identify the damage on a benchmark building investigated by Lin et al. (2005). Cross-correlation analysis (CCA) of recorded random vibration data in the test section of a large cavitation channel has been implemented to capture deterministic time signatures, which lead to the extraction of structural response by Sabra et al. (2007). In 2007, the CCA employed to detect the damage in a laboratory composite beam under random excitation. To this end, CCF

Received: Jun. 21, 2019 | Accepted: Nov. 04, 2020

Peer Review History: Double Blind Peer Review.

Section Editor: Valdeer Steffen Junior

This is an open access article distributed under the terms of the Creative Commons license. 
amplitude vector of structural response was defined. (Yang et al. 2007; Zhu et al. 2010). Wang et al. (2010) developed the CCF between vibration velocity and acceleration responses as a structural damage detection technique.

Zhang et al. (2014) have proposed several new correlation function-based damage detection methods (autocorrelation function at maximum point value vector [AMV] method, the maximum value of the CCF [CMV] method and zero value of the CCF [CZV] method) that require no reference point. The damage detection results show that the proposed three new methods can not only effectively locate the damage even when noise exists, but also require no reference point compared to CorV/inner product vector (IPV) method, among which the AMV method is the best. Zhang and Schmidt (2014) studied the sensitivity of an auto correlation function-based damage index. They showed that the normalized AMV has a better recognizability. Wang et al. (2011) demonstrated that the IPV can be measured by the inner product of the time domain vibration responses of each measurement points under band pass white noise excitation or white noise excitation, and the computational cost is highly reduced (Wang et al. 2011; Wang and Yang 2012).

Damage-induced dynamic characteristics may be entirely masked by variations in the dynamic characteristics because of differences in the environmental temperature (Xu et al. 2017). Without understanding temperature effects, false structural condition identification may occur. Some studies have showed temperature variations effects in the structural vibration properties (Mohammadi Esfarjani et al. 2017; Mohammadi Esfarjani, 2020; Xia et al. 2012). In recent years, research on assessing the effect of environment factors, such as the temperature on vibration-based damage detection (VBDD) techniques using FE methods, has become very popular.

The IPV method is a new VBDD technique that has been introduced in recent years. Previous researches showed that the IPV method is able to detect internal and surface structural damages (Mohammadi Esfarjani and Salehi 2016a,b,c; 2017; 2020). The IPV method is more sensitive to damage than CMV and CZV methods (Zhang et al. 2014). Also, the IPV can be calculated by displacement responses, velocity responses and acceleration response. None of the previous studies considered changes in the environmental conditions such as temperature changes on the damage detection capability of the IPV method. This paper is the first investigation on the effect of changes in the temperature on the IPV method performance. In this study, an Airbus A320 slat-track was investigated. Given the maximum and minimum temperatures in the coldest and hottest airports in the world and assuming the aircraft is traveling between these regions, a temperature range of -70 to $70{ }^{\circ} \mathrm{C}$ is sufficient for the present study. However, in this paper, a greater temperature range was selected to achieve wider and more accurate judgments about the changing temperature effect on the IPV method. The simulated structure was evaluated under a temperature between of -73 and $260^{\circ} \mathrm{C}$. The vibration responses were obtained via numerical simulation with the ABAQUS (commercial Finite Element software). The IPV was calculated from vibration responses under band pass white noise excitation using a developed MATLAB code.

\section{THEORY OF IPV AND TEMPERATURE CHANGES EFFECT ON IT}

In this section, theory of IPV is introduced and then temperature changes effect on the theory of IPV are presented.

\section{Theory of the IPV}

To calculate the IPV, firstly, response measurements including displacement, velocity and acceleration are fulfilled. Then, the inner product of the time domain vibration responses of each measurement point under white noise excitation or band pass white noise excitation is computed (Eqs. 1-3) (Wang et al. 2010, 2011; Zhang and Schmidt 2014).

$$
\begin{aligned}
& R_{I P V, j}^{\mathrm{dis}}=\left[R_{x_{1} x_{j}}(0), R_{x_{2} x_{j}}(0), \ldots, R_{x_{p} x_{j}}(0)\right]^{\mathrm{T}}=\sum_{r=1}^{n} \zeta_{j, r}^{\mathrm{dis}} \varphi_{r} \\
& R_{I P V, j}^{\mathrm{vel}}=\left[R_{\dot{x}_{1} \dot{x_{J}}}(0), R_{\dot{x}_{2} \dot{x}_{J}}(0), \ldots, R_{\dot{x}_{p} \dot{x_{j}}}(0)\right]^{\mathrm{T}}=\sum_{r=1}^{n} \zeta_{j, r}^{\mathrm{vel}} \varphi_{r} \\
& R_{I P V, j}^{\mathrm{acc}}=\left[R_{\ddot{x}_{1} \ddot{x}_{J}}(0), R_{\ddot{x}_{2} \ddot{x}_{J}}(0), \ldots, R_{\ddot{x}_{p} \ddot{x}_{J}}(0)\right]^{\mathrm{T}}=\sum_{r=1}^{n} \zeta_{j, r}^{\mathrm{acc}} \varphi_{r}
\end{aligned}
$$


where $\left|\zeta_{j, r}^{\mathrm{dis}}\right|,\left|\zeta_{j, r}^{\mathrm{vel}}\right|$ and $\left|\zeta_{j, r}^{\mathrm{acc}}\right|$ are weighting factors. A local damage index determined by the difference between the IPVs of the undamaged and damaged structures is used to reduce the effect of measurement noise (Eq. 4) (Wang et al. 2010; 2011; Wang and Yang 2013):

$$
D_{\mathrm{IPV}, j}=R_{\mathrm{IPV}, j}^{\mathrm{d}}-R_{\mathrm{IPV}, j}^{\mathrm{u}}
$$

where $R_{\mathrm{IPV}, j}^{\mathrm{u}}$ and $R_{\mathrm{IPV}, j}^{\mathrm{d}}$ coefficients show the $i$ th element in the IPVs of the healthy and damaged structures, respectively. Afterward, the damage index is determined as $D_{\mathrm{IPV}, j}=\left\{D_{\mathrm{IPV}, 1}, D_{\mathrm{IPV}, 2}, \ldots, D_{\mathrm{IPV}, \mathrm{N}_{\mathrm{m}}}\right\}$. Since the damage detection is performed using local maximum of the damage index, a threshold for categorizing the undamaged and damaged structure should be selected (Eqs. 5 and 6) (Wang et al. 2011; Wang and Yang 2012).

$$
\begin{gathered}
t_{h}=\mu_{\mathrm{D}}+\alpha_{C} \sigma_{\mathrm{D}} \\
t_{l}=\mu_{\mathrm{D}}-\alpha_{C} \sigma_{\mathrm{D}}
\end{gathered}
$$

where $\mu_{\mathrm{d}}$ is the mean value of $D_{\mathrm{IPV}}\left(\right.$ or $\left.D_{\mathrm{IPV}}^{\text {or }} D_{\mathrm{IPV}}^{\ddot{*}}\right), \sigma_{\mathrm{D}}$ is the standard deviation of $D_{\mathrm{IPV}}\left(\right.$ or $D_{\mathrm{IPV}}^{*}$ or $\left.D_{\mathrm{IPV}}^{*}\right)$ and $\alpha_{\mathrm{c}}$ a coefficient corresponding to a confidence interval

If the elements of $D_{\text {IPV }}$ (or $D_{\text {IPV }}^{\text {or }} D_{\text {IPV }}^{.}$), located in the area between $t_{1}$ and $t_{\mathrm{h}}$, it shows that the structure is undamaged; otherwise, the structure is damaged.

\section{Temperature changes effect on the theory of IPV}

Under normal operating conditions, the operation of a civil aircraft is usually considered to range from -40 to $60^{\circ} \mathrm{C}$. Equation 7 can be used to determine the effect of temperature on properties when considering a linear approximation for changes in properties with temperature (Di Scalea and Salamone 2008).

$$
P(T)=P\left(T_{0}\right)+\frac{\partial P(T)}{\partial T} \Delta T
$$

where $P$ represents one of the properties, $T_{0}$ the ambient temperature $20^{\circ} \mathrm{C}, T$ the generic temperature and $\frac{\partial P(T)}{\partial T}$ the sensitivity to temperature.

\section{SIMULATION VERIFICATION OF THE IPV METHOD}

In recent years, the FE method has been widely applied for SHM based VBDD methods instead of experimental testing, because experimental testing is a costly and time-consuming procedure (Moragaspitiya et al. 2013). In this paper, a simulation model is employed to study the effect of temperature on IPV technique performance. To verify this simulation model, we referred to our earlier experimental research. As shown in Fig. 1, the pipe has a dimension of $60 \mathrm{~cm}$ in length, $1.5 \mathrm{~cm}$ in outer diameter and $1 \mathrm{~mm}$ in wall thickness. It was modelled through the FE software ABAQUS (Fig. 1). The findings from simulation model are compared with the empirical results that showed good correspondence.

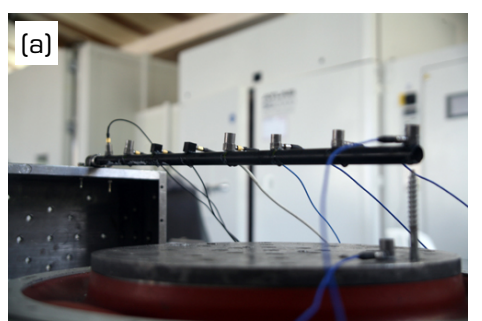

(b)

Figure 1. The IPV test. (a) Experimental test; (b) A FE model with ABAQUS. 


\section{NUMERICAL SIMULATION}

In this section, the ability of the IPV method to determine the existence and position of a longitudinal crack on an Airbus 320 slat-track with a longitudinal crack is assessed. Then, sensitivity analysis of the IPV method to temperature changes is evaluated. The location of the A320 slat-track in the airplane wing is shown in Fig. 2 (Pitropakis et al. 2012). In practice, there are almost no problems reported with these kinds of slat tracks. There is, however, a growing interest in SHM for different reasons (Pfeiffer et al. 2007).
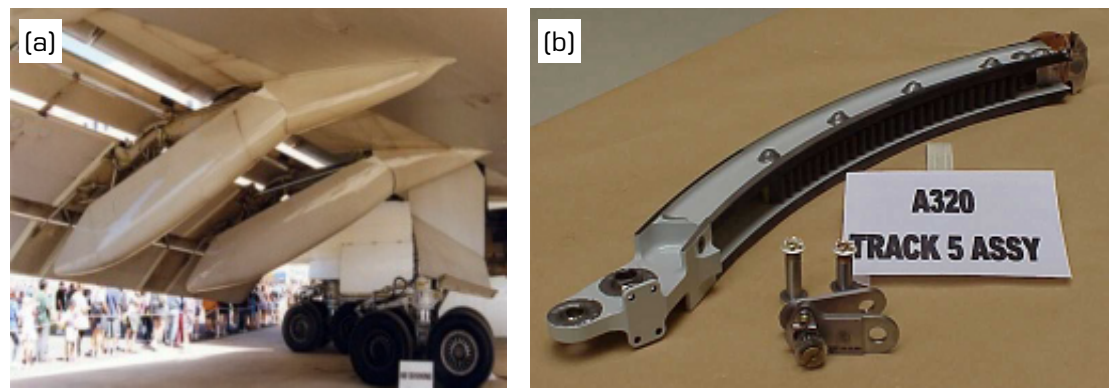

Figure 2. Slat-track of Airbus A320. (a) Position in airplane wing; (b) A dismounted sample.

\section{Detecting damage}

The vibration responses are obtained via numerical simulation with the commercial FE package ABAQUS. Vibration responses are processed in MATLAB to achieve some meaningful damage detection features (Fig. 3). In the next section, the A320 slat-track with a longitudinal crack is considered.

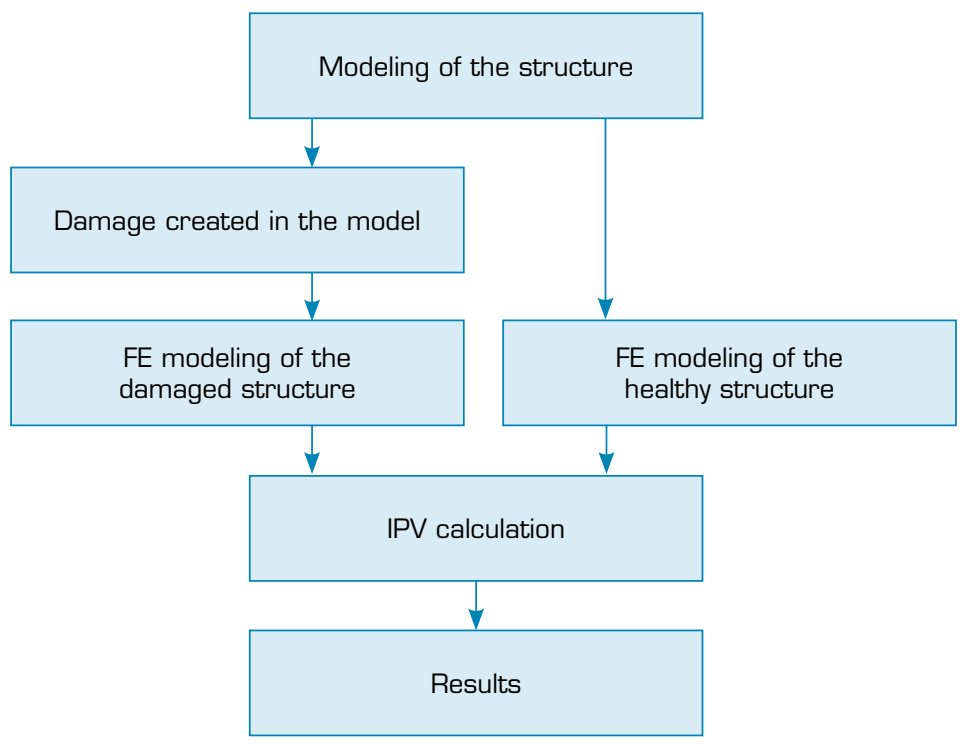

Figure 3. Flowchart of the method used for detecting damage.

\section{Modeling and analysis of a healthy airbus $\mathrm{A} 320$ slat-track}

The length of A320 slat-track is $64 \mathrm{~cm}$. The material properties are listed in Table 1. Firstly, a FE model of the A320 slat-track was produced using ABAQUS. The clamped end of the beam has no rotation or displacement in any direction. The excitation force is applied in the direction of the positive y-axis at its mid-point of the free side (Fig. 4). Multiple runs showed that the type of mesh has a nonsignificant effect on results, while free meshing is more suitable on the damaged section. Thus, free meshing with triangular and tetrahedral elements is utilized (Fig. 5). Then, the model was analysed with modal dynamic step (Fig. 6). Finally, desired data, including displacement, velocity and acceleration responses for 16 points, were obtained from the results. 
Table 1. Material properties for maraging steel (grade 250)

\begin{tabular}{cccc}
\hline Material & $\begin{array}{c}\text { Density }[\rho] \\
{[\mathrm{g} / \mathrm{cc}]}\end{array}$ & Poisson's ratio $[\mathrm{v}$ ] & $\begin{array}{c}\text { Modulus of elasticity }[\mathrm{E}] \\
\text { [Gpa] }\end{array}$ \\
\hline Maraging steel (grade 250) & 8.02 & 0.3 & 190 \\
\hline
\end{tabular}

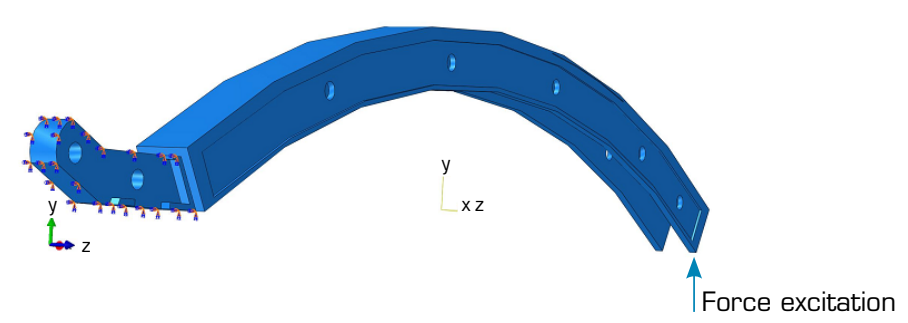

Figure 4. Boundary condition and force excitation in the modal.

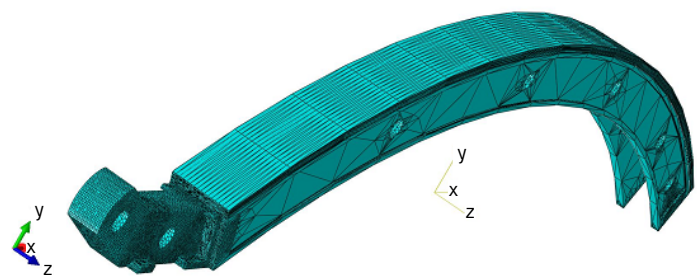

Figure 5. Using free meshing technique with linear tetrahedral and hexahedral elements in simulation modelling.

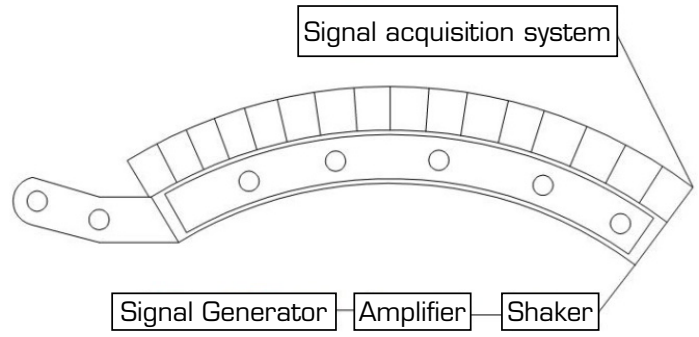

Figure 6. Scheme of the test and the position of nodes that are measured on the healthy structure.

\section{Simulation modelling and analysis of damaged structure}

At this stage, the longitudinal crack was created in the model (Fig. 7). The size of the longitudinal crack is $21 \times 3.2 \times 0.01 \mathrm{~mm}$, and it is placed at $37 \mathrm{~cm}$ from the fixed end. Finally, the damaged model was analysed in the modal dynamic step. Displacement, velocity and acceleration responses for 16 points were obtained from the results.

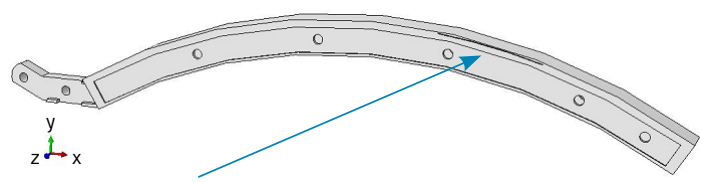

Figure 7. A longitudinal crack in the A320 slat-track created in the model.

\section{RESULTS AND DISCUSSION}

The results of the IPV method for the detection of a longitudinal crack in an Airbus 320 slot-track are shown in Figs. 8-10. It is necessary to determine the confidence interval factor (CIF) in the IPV method. In this paper, an accurate value for the CIF was 
measured using optimized model for structural health monitoring based on IPV. As shown in Figs. $8-10$, when the CIF $\left(\alpha_{c}\right)$ is chosen 1.2 or 1.5, the IPV graph is not confluent with the line threshold, which means that classifying damaged and undamaged structures is not possible. When the CIF is 1.8, damage can be detected. Figures 8 and 9 shows the IPV calculated from displacement and velocity responses indicate that damage is detectable between 39 and $45 \mathrm{~cm}$ from the fulcrum of the A320 slat-track. Moreover, as shown in Fig. 10, the line threshold does make confluence with the IPV graph $45 \mathrm{~cm}$ from the fulcrum of the A320 slat-track; in other words, the IPV calculated from the acceleration response indicates that damage is detectable at this distance. It is evident from Figs. 8-10, that the IPV method is successful in detecting a longitudinal crack in the Airbus 320 slot-track, as well as its location, with close approximation.

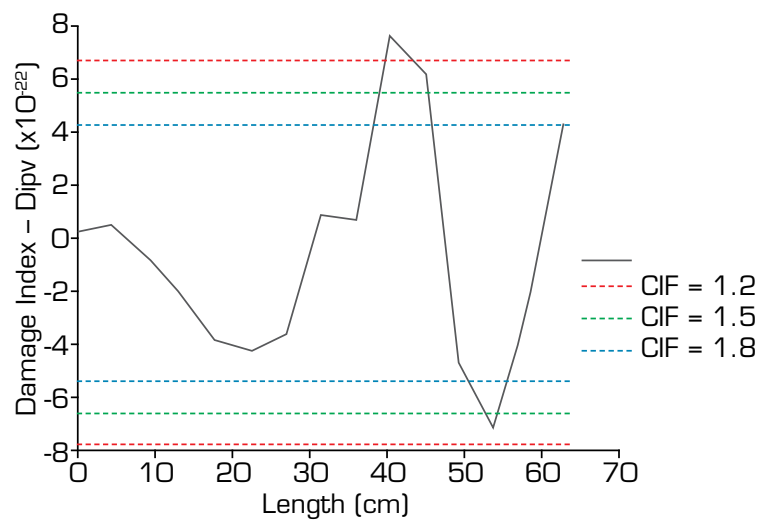

Figure 8. Detecting damage for a longitudinal crack in the A320 slat-track using displacement responses.

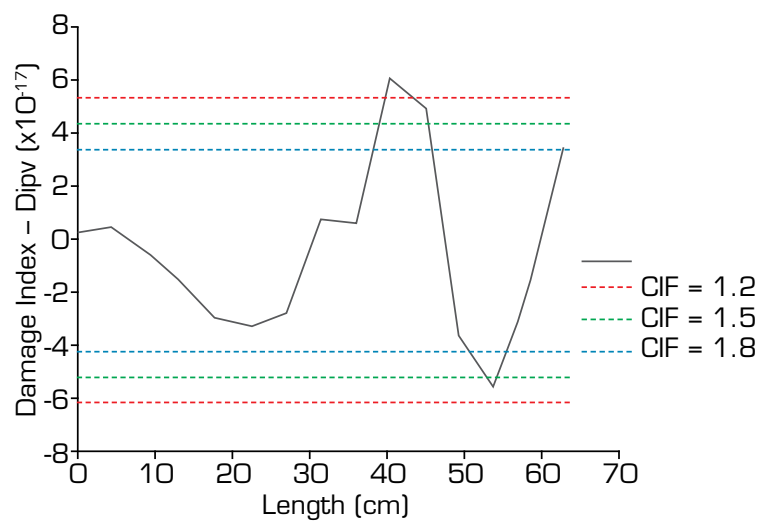

Figure 9. Detecting damage for a longitudinal crack in the A320 slat-track using velocity responses.

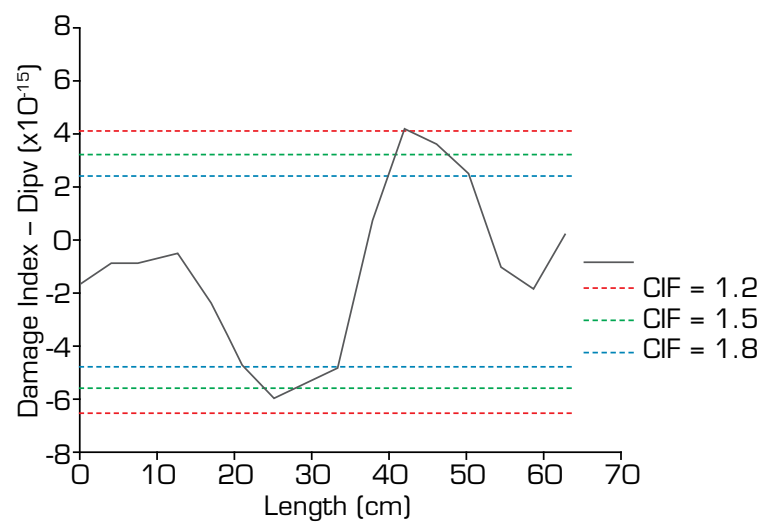

Figure 10. Detecting damage for a longitudinal crack in the A320 slat-track using acceleration responses. 


\section{The sensitivity of the IPV method to environmental noise}

Receiver operating characteristic (ROC) curve is one technique that enables the statistical evaluation of the errors related with false detection. A ROC curve is a two-dimensional graphic in which the true positive rate (TPr) is plotted in the $y$ axis and the false positive rate (FPr) is plotted in the $\mathrm{x}$-axis and the graphic demonstrates relative trade-offs between these benefits and costs, respectively (Neves et al. 2018). Figure 11 shows the ROC curves of four classification models, signal-to-noise ratio ( $\mathrm{SNR}$ ) $=15 \mathrm{~dB}$, $\mathrm{SNR}=20 \mathrm{~dB}, \mathrm{SNR}=25 \mathrm{~dB}$ and $\mathrm{SNR}=30 \mathrm{~dB}$. The diagonal line representing random guessing is also shown. Thus, the closer the ROC curve of a model is to the diagonal line, the less accurate the model (Han et al. 2011). As shown in Fig. 11, SNR = 15 dB is less accurate.

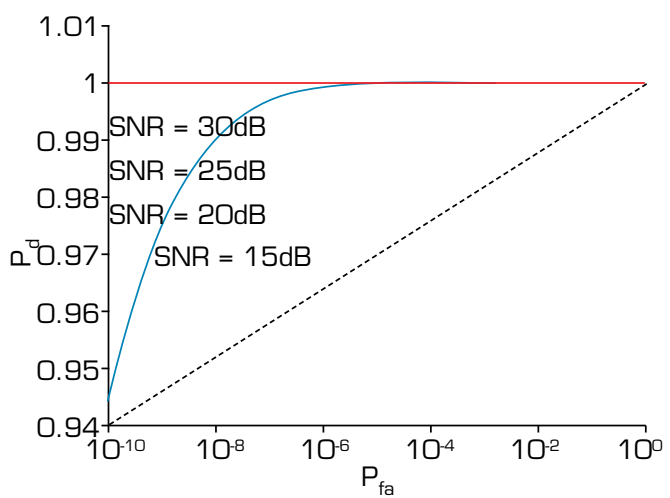

Figure 11. The ROC curves of four classification models, $S N R=15,20,25$ and $30 \mathrm{~dB}$.

As SNR = $15 \mathrm{~dB}$ is less accurate model, it is necessary to evaluate the performance of the IPV method able to detect the damage despite the environmental noise. To achieve that, the structural dynamic vibrational responses for 15 points were obtained for healthy and damaged from the simulation with environmental noise. Finally, the damage was identified by the IPV method (Fig. 12). As shown in Fig. 12, the IPV method is able to detect the damage despite the environmental noise.

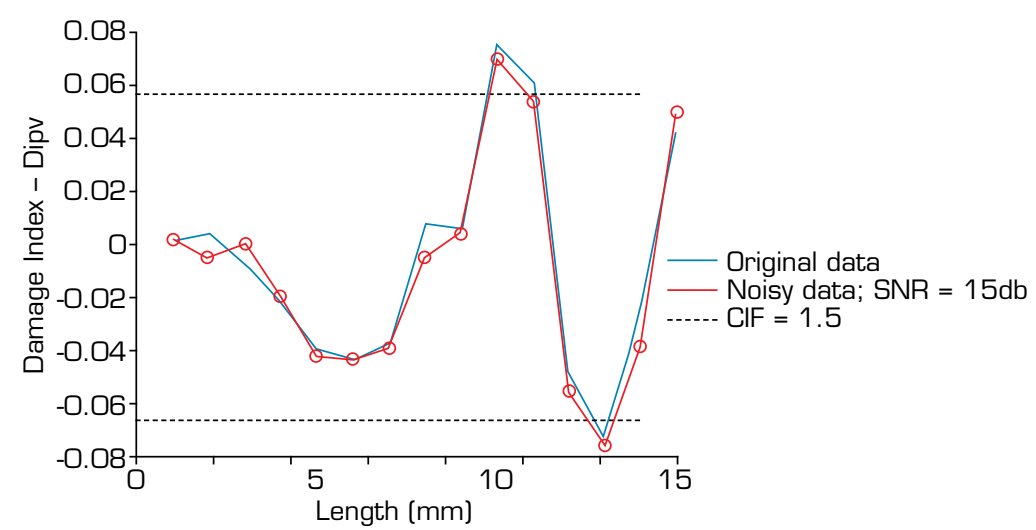

Figure 12. Detecting damage for a longitudinal crack in the A320 slat-track using displacement responses with $\mathrm{SNR}=15 \mathrm{~dB}$.

\section{Effect of temperature on the IPV method performance}

In this section, sensitivity analysis of the IPV method to temperature changes is addressed. The vibration responses are obtained via numerical simulation with the commercial FE package ABAQUS. Then, the IPV concerning vibration response to band pass white noise excitation can be obtained using MATLAB. To this purpose, an A320 slat-track, which is made of stainless steel 304, is evaluated using the IPV method at ambient temperature of $21^{\circ} \mathrm{C}$. The results of analysis of the healthy structure obtained by the IPV method at a temperature of $21^{\circ} \mathrm{C}$ will be considered as the reference temperature and initial situation $\left(R_{I P V, j}^{d}\right)$ for the inspection process. Then, the healthy structure is evaluated in a temperature between of -73 and $260^{\circ} \mathrm{C}$. The results of analysis of a healthy structure obtained by the IPV method in a temperature between of -73 and $260{ }^{\circ} \mathrm{C}$ is selected as the secondary status 
$\left(D_{I P V, j}\right)$ for the inspection process. Finally, local damage index $\left(D_{I P V, j}\right)$ is calculated by the IPV method. If the IPV method is not sensitive to temperature change, then the results should not show any damage in structure, in other words, the slope of $D_{I P V}$ chart is zero $\left(D_{I P V, j}=R_{I P V, j}^{d}-R_{I P V, j}^{u}=0\right)$. A flowchart of the method used to investigate temperature changes effect on the IPV method is shown in Fig. 13.

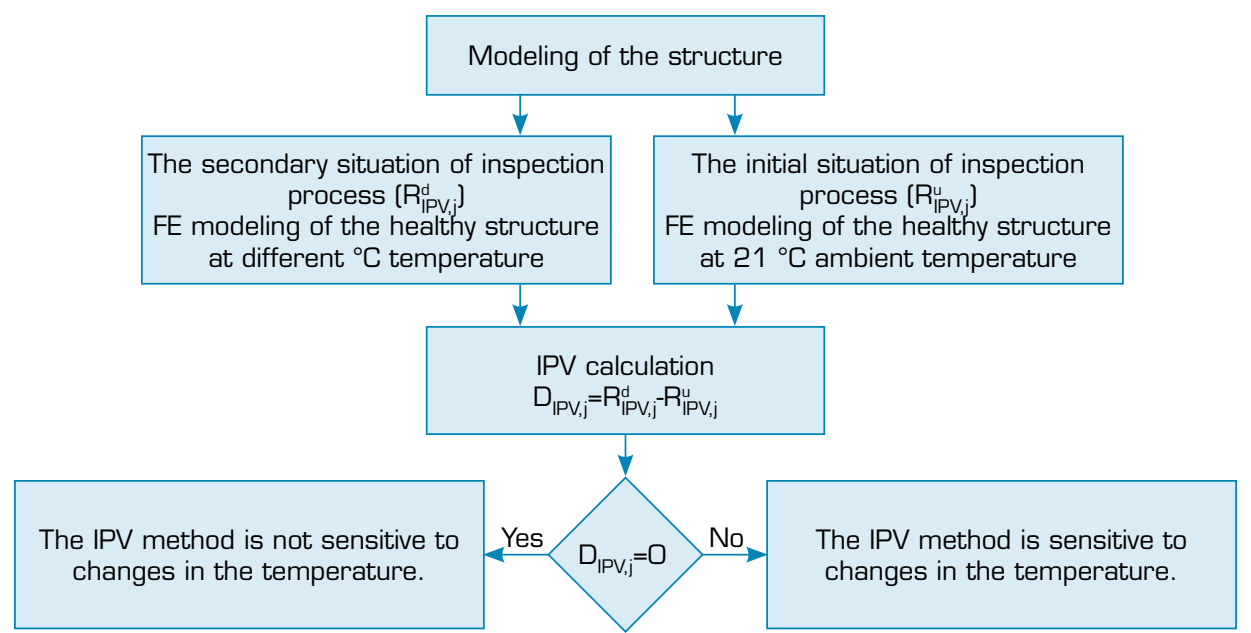

Figure 13. Flowchart of the method used to investigate temperature changes effect on the IPV method.

\section{Investigation of the ipv method under constant temperature}

If the structure is healthy at the secondary status and the temperature of the structure is the same for the initial and secondary statuses, it is expected that the results do not show any damage in the structure. In other words, the slope of $D_{I P V}$ chart is zero $\left(D_{I P V, j}=R_{I P V, j}^{d}-R_{I P V, j}^{u}=0\right)$. As shown in Fig. 14, if the temperature is constant, the slope of $D_{I P V}$ chart is zero.

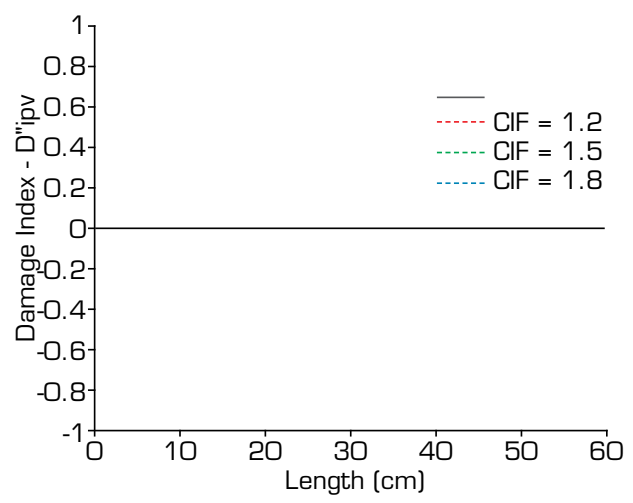

Figure 14. Result of SHM of the A320 slot-track of a healthy using acceleration responses, the temperature of structure is $21\left({ }^{\circ} \mathrm{C}\right)$ for the initial and secondary statuses of inspection.

\section{Inner product vector method performance evaluation}

In this step, the structure is healthy at the secondary status and the temperature of the structure is altered for the secondary status. The structure is evaluated at temperatures $-73,149$ and $260^{\circ} \mathrm{C}$. Obtained results are shown in Figs. 15-17. The optimization process is applied to obtain an accurate value for the CIF. As previously stated, the threshold value for CIF ( $\left.\alpha_{c}\right)$ is set to 1.8 for stainless steel of type 304. As shown in Figs. 15-17, the slope of $D_{I P V}$ chart is not zero $\left(D_{I P V, j}=R_{I P V, j}^{d}-R_{I P V, j}^{u} \neq 0\right)$. These figures show that the elements of $D_{I P V}$ fall out the region between $t_{1}$ and $t_{\mathrm{k}}$, accordingly. It can be concluded that the structure is damaged. So, change of temperature causes the IPV method to show spurious defect in structure. This can be explained by temperature changes affecting material properties, such as Young's modulus. The Young's modulus $(E)$ is a measure of stiffness, 
so temperature change reduces the structural stiffness. Accordingly, in the IPV method, damage is a reduction in the structural stiffness, so the IPV method shows spurious defect in structure with changes in temperature. The findings of this study show that the IPV method is sensitive to changes in temperature, so the temperature of the structure should be the same for the initial and secondary statuses of SHM when using the IPV method.

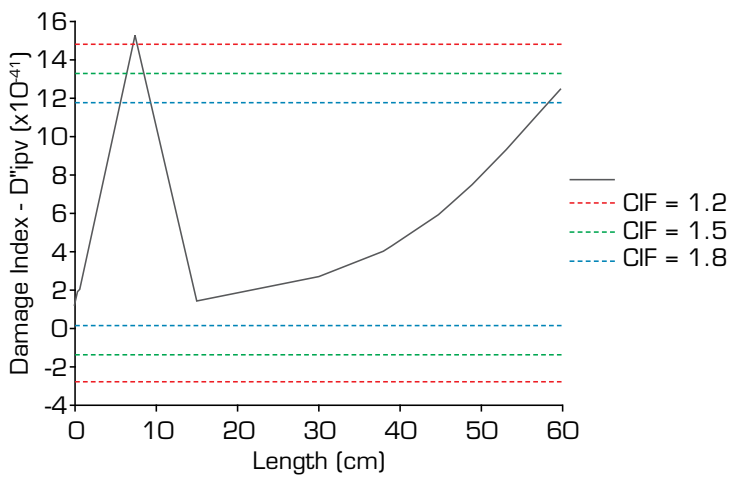

Figure 15. Result of SHM of the healthy A 320 slot-track using acceleration responses. The temperature of structure is $21{ }^{\circ} \mathrm{C}$ for the initial and $260^{\circ} \mathrm{C}$ for secondary statuses of inspection.

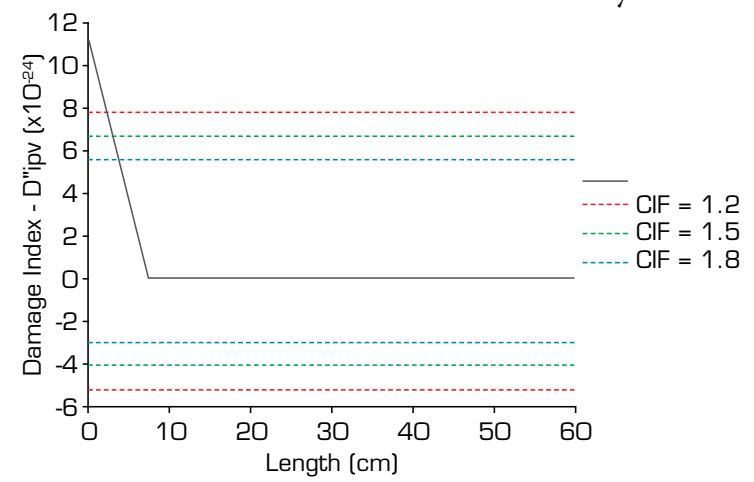

Figure 16. Result of SHM of the healthy A 320 slot-track using acceleration responses. The temperature of structure is $21^{\circ} \mathrm{C}$ for the initial and $149^{\circ} \mathrm{C}$ for secondary statuses of inspection.

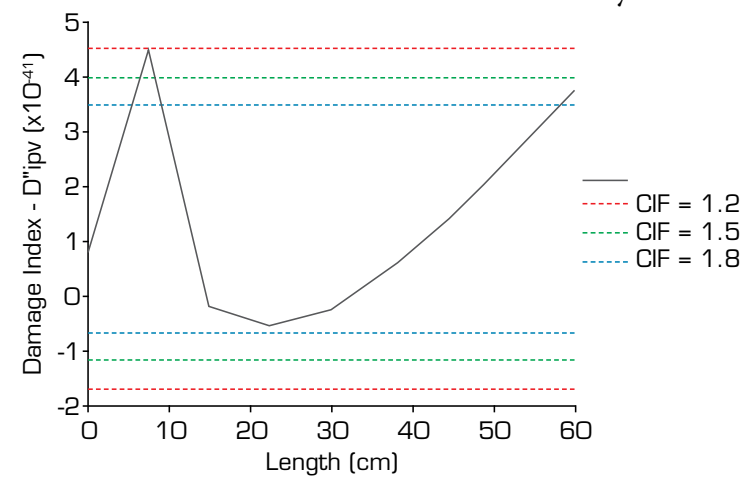

Figure 17. Result of SHM of the healthy A 320 slot-track using acceleration responses. The temperature of structure is $21^{\circ} \mathrm{C}$ for the initial and $-73{ }^{\circ} \mathrm{C}$ for secondary statuses of inspection.

\section{CONCLUSION}

The purpose of the present paper was to study the effect of temperature change on the theory of IPV. Inner product vector method can be used to detect structural damage. Firstly, this study evaluates the IPV method ability to detect damage in an Airbus A320 slat-track, assessed by a longitudinal crack. The findings of this study show that the IPV method is succeeded 
in detecting defect in the structure, as well as its location, with close approximation. Then, the sensitivity of the IPV method to environmental noise was investigated. The results show that the IPV method is able to detect the damage despite the environmental noise. Finally, the Airbus A320 slat-track was investigated for the effect of changes in temperature on the IPV method, evaluated over a temperature between of -73 and $260^{\circ} \mathrm{C}$. This is the first attempt to study the effect of temperature changes on the IPV method ability to detect damage. The results from the IPV method show a spurious defect in the structure with temperature change; therefore, the IPV method is temperature-sensitive. Also, this study highlighted the importance of applying simulation methods to develop VBDD techniques, especially for evaluating the effect of changes in environmental temperature when the structure is complex.

\section{AUTHORS' CONTRIBUTION}

Conceptualization: Mohammadi Esfarjani S; Methodology: Mohammadi Esfarjani S; Investigation: Mohammadi Esfarjani S; Writing - Original Draft: Mohammadi Esfarjani S; Writing - Review and Editing: Mohammadi Esfarjani $\mathrm{S}$ and Salehi M.

\section{DATA AVAILABILITY STATEMENT}

Data will be available upon request

\section{FUNDING}

Not applicable.

\section{ACKNOWLEDGEMENTS}

Not applicable.

\section{REFERENCES}

Di Scalea FL, Salamone S (2008) Temperature effects in ultrasonic Lamb wave structural health monitoring systems. J Acoust Soc Am 124(1):161-174. https://doi.org/10.1121/1.2932071

Farrar CR, James III GH (1997) System Identification from Ambient Vibration Measurements on a Bridge. J Sound Vib 205(1):1-18. https://doi.org/10.1006/jsvi.1997.0977

Han J, Kamber M, Pei J (2011) Data Mining: Concepts and Techniques. Burlington: Morgan Kaufmann.

Labib A, Kennedy D, Featherston C (2014a) Free vibration analysis of beams and frames with multiple cracks for damage detection. J Sound Vib 333(20):4991-5003. https://doi.org/10.1016/j.jsv.2014.05.015

Lin S, Yang JN, Zhou L (2005) Damage identification of a benchmark building for structural health monitoring. Smart Mater Struct 14(3):S162. https://doi.org/10.1088/0964-1726/14/3/019 
Medeiros R, Sartorato M, Marques FD, Vandepitte D, Tita V (2013) Vibration-based damage identification applied for composite plate: Experimental analyses. Paper presented 22nd International Congress of Mechanical Engineering (COBEM 2013). COBEM; Ribeirão Preto, São Paulo, Brazil.

Mohammadi Esfarjani S, Salehi M (2016a) Evaluation of the damage detection capability of inner product vector for LOP and LOSWF defects in V groove weld. Modares Mech Eng 16(6):7-16.

Mohammadi Esfarjani S, Salehi M (2016b) Damage identification in aluminium T3-2024 alloy via cross correlation functions. Paper presented 15th International Conference of Iranian Aerospace. Tehran, Iran.

Mohammadi Esfarjani S, Salehi M (2016c) Detection of Metallic Impurities in Alloys Using the IPV and AMV Methods. Romanian J Acoust Vib 13(2):131-137.

Mohammadi Esfarjani S, Salehi M (2017) Optimization the inner product vector method and its application to structural health monitoring. J Vibroeng 19(4):2578-2585. https://doi.org/10.21595/jve.2017.18062

Mohammadi Esfarjani S, Salehi M (2020) Inspection above ground pipeline using vibration responses, J Pipeline Syst Eng Pract 11(3): 04020021 https://doi.org/10.1061/(ASCE)PS.1949-1204.0000463

Mohammadi Esfarjani S, Salehi M, Ghassemi A. (2017) Effect of the Multiple Damages and Temperature Changes on the Natural Frequency. J Theor App Mech-Pol 55(3):813-822. https://doi.org/10.15632/jtam-pl.55.3.813

Mohammadi Esfarjani S (2020) Evaluation of effect changing temperature on lamb-wave based structural health monitoring. J Mech Energy Eng 3(4):329-336. https://doi.org/10.30464/jmee.2019.3.4.329

Moragaspitiya PHN, Thambiratnam DP, Perera NJ, Chan THT (2013) Development of a vibration based method to update axial shortening of vertical load bearing elements in reinforced concrete buildings. Eng Struct 46:49-61. https://doi. org/10.1016/j.engstruct.2012.07.010

Neves AC, González I, Leander J, Karoumi R (2018) A New Approach to Damage Detection in Bridges Using Machine Learning. In: Conte JP, Astroza R, Benzoni G, Feltrin G, Loh KJ, Moaveni B, editors. Experimental Vibration Analysis for Civil Structures. Lecture Notes in Civil Engineering Vol 5. Cham: Springer. https://doi.org/10.1007/978-3-319-67443-8_5

Ni P, Xia Y, Law S, Zhu S (2014b) Structural Damage Detection Using Auto/Cross-Correlation Functions Under Multiple Unknown Excitations. Int J Struct Stab Dyn 14(5):1440006. https://doi.org/10.1142/S0219455414400069

Pfeiffer H, De Baere D, Fransens F, Van Der Linden G, Wevers M (2007) Structural health monitoring of Slat Tracks using transient ultrasonic waves. Paper presented EU Project Meeting on Aircraft Integrated Structural Health Assessment. AISHA; Leuven, Belgium.

Pitropakis I, Pfeifer H, Gesang T, Janssens S, Wevers M (2012) Crack Detection on an Airbus A320 Slat-Track and on Aluminium 2024-T3 Plates using Crack Propagation Gauges. Paper presented 18th World Conference on Nondestructive Testing. WCNDT; Durban, South Africa.

Sabra KG, Winkel ES, Bourgoyne DA, Elbing BR, Ceccio SL, Perlin M, Dowling DR (2007) Using cross correlations of turbulent flow-induced ambient vibrations to estimate the structural impulse response. Application to structural health monitoring. J. Acoust. Soc. Am 121(4):1987-1995. https://doi.org/10.1121/1.2710463

Wang L, Yang ZC (2012) Structural Damage Detection Using Inner Product Vector and Low Pass Filter Technique. Applied Mechanics and Materials 204-208:2942-2946. https://doi.org/10.4028/www.scientific.net/AMM.204-208.2942

Wang L, Yang Z, Waters TP (2010) Structural damage detection using cross correlation functions of vibration response. J Sound Vib 329(24):5070-5086, https://doi.org/10.1016/j.jsv.2010.06.020 
Wang L, Yang Z, Waters TP, Zhang M (2011), Theory of inner product vector and its application to multi-location damage detection. J Phys: Conf Ser 305:012003. https://doi.org/10.1088/1742-6596/305/1/012003

Xia Y, Chen B, Weng S, Ni Y-Q, Xu Y-L (2012) Temperature effect on vibration properties of civil structures: a literature review and case studies. J Civil Struct Health Monit 2:29-46. https://doi.org/10.1007/s13349-011-0015-7

Xu Z-D, Wu Z (2017) Simulation of the Effect of Temperature Variation on Damage Detection in a Long-span Cable-stayed Bridge. Struct Health Monit 6(3):177-189. https://doi.org/10.1177/1475921707081107

Yang, Z. C., Yu, Z. F., Sun, H. (2007) On the cross correlation function amplitude vector and its application to structural damage detection. Mech Syst Signal Process 21(7):2918-2932, https://doi.org/10.1016/j.ymssp.2007.03.004

Zhang M, Schmidt R (2014) Sensitivity analysis of an auto-correlation-function-based damage index and its application in structural damage detection. J Sound Vib 333(26): 7352-7363. https://doi.org/10.1016/j.jsv.2014.08.020

Zhang M, Schmidt R, Markert B (2014) Structural damage detection methods based on the correlation functions. Paper presented $9^{\text {th }}$ International Conference on Structural Dynamics. EURODYN; Porto, Portugal.

Zhu D, Yi X, Wang Y, Sabra K (2010) Structural damage detection through cross correlation analysis of mobile sensing data. Paper presented $5^{\text {th }}$ World Conference on Structural Control and Monitoring. 5WCSCM; Tokyo, Japan. 